\title{
Indications for GABA-Immunoreactive Axo-Axonic Contacts on the Intraspinal Arborization of a Ib Fiber in Cat: A Confocal Microscope Study
}

\author{
Boris Lamotte d'Incamps, ${ }^{1}$ Josette Destombes, ${ }^{1}$ Danièle Thiesson, ${ }^{1}$ Raymond Hellio, ${ }^{2}$ Xavier Lasserre, ${ }^{1}$ \\ Nezha Kouchtir-Devanne, ${ }^{1}$ Léna Jami, ${ }^{1}$ and Daniel Zytnicki ${ }^{1}$ \\ ${ }^{1}$ Centre National de la Recherche Scientifique Unité de Recherche Associée 1448, Université René Descartes, 75270 \\ Paris Cedex 06, France, and 2Service de Microscopie Confocale, Institut Pasteur, 75724 Paris Cedex 15, France
}

Confocal microscopy was used to detect GABAimmunoreactive axo-axonic appositions, indicating possible synaptic contacts, on Ib fiber terminals in the lumbosacral spinal cord. A lb fiber from posterior biceps-semitendinosus muscles was labeled by intra-axonal ejection of tetramethylrhodamine dextran (red), and serial sections of S1-L7 spinal cord segments were processed for GABA immunocytochemistry revealed by fluorescein isothiocynate (green). Appositions between GABA-immunoreactive structures and the labeled fiber appeared as yellow spots because of the presence of both fluorochromes in small volumes $\left(0.3 * 0.3 * 0.5 \mu \mathrm{m}^{3}\right)$ of tissue. These spots were identified as probable axo-axonic contacts when: (1) they were observed in two to four serial confocal planes, indicating that they did not occur by chance; and (2) their sizes, shapes, and locations were similar to those of axo-axonic contacts found on la terminals, known to bear presynaptic boutons, and resembled the axo-axonic synapses described in electron microscope studies of $\mathrm{lb}$ boutons in Clarke's column. A total of 59 presumed axo-axonic contacts was observed on two lb collaterals, representing an estimated $20 \%$ of the total complement. In a three-dimensional reconstruction of one collateral, they were mostly located in terminal positions, and some branches bore more contacts than others. Such differential distribution could not result from chance appositions between GABAergic structures and Ib arborization and further supported the identification of axo-axonic contacts. Segmental lb collaterals bear axo-axonic synapses that might ensure differential funneling of information toward different targets.

Key words: Ib afferent fiber; GABAergic axo-axonic contacts; presynaptic inhibition; motor control; mammals; confocal microscopy
The intraspinal portion of Ib afferent fibers from ankle extensor muscles displays primary afferent depolarizations (PADs) during contractions, indicating that contraction-induced afferent inputs can elicit presynaptic inhibition of Ib terminals (Lafleur et al., 1992).

Most of the available data on presynaptic inhibition of group I afferents in the spinal cord were obtained from studies of Ia fibers. It was established that GABA-immunoreactive axo-axonic synapses, contacting the intraspinal terminals of these fibers, are the morphological substrate of presynaptic inhibition (Rudomin, 1990). They act primarily through activation of $\mathrm{GABA}_{\mathrm{A}}$ receptors, possibly with a contribution of $\mathrm{GABA}_{\mathrm{B}}$ receptors (Stuart and Redman, 1992; Curtis et al., 1997; see also Eccles et al., 1963; Rudomin et al., 1983). Electron microscope studies provided ultrastructural evidence for presynaptic boutons ( $\mathrm{P}$ boutons) on en passant or terminal Ia boutons contacting motoneurons in lamina IX (Conradi et al., 1983; Fyffe and Light, 1984; Pierce and Mendell, 1993) or neurons in lamina VI in which P boutons were found to be GABA-immunopositive (Maxwell et al., 1990).

Received April 27, 1998; revised August 18, 1998; accepted Sept. 21, 1998.

This work was supported by the Association Française Contre les Myopathies (MNM 1996), Groupement d'Intérêt Scientifique "Sciences de la Cognition" (CNA46), and DGA-DRET (95062). We thank Pr. Yves Laporte, Dr. Constantino Sotelo, and Dr. Claude Meunier for critical reading of this manuscript and helpful suggestions.

Correspondence should be addressed to Dr. D. Zytnicki, Centre National de la Recherche Scientifique Unité de Recherche Associée 1448, Université René Descartes, 45 rue des Saints-Pères, 75270 Paris Cedex 06, France.

Copyright (C) 1998 Society for Neuroscience $\quad 0270-6474 / 98 / 1810030-07 \$ 05.00 / 0$
In the case of Ib fibers, presynaptic contacts have been observed on boutons located in Clarke's column (Walmsley et al., 1987), but they had not been demonstrated on segmental terminals located more caudally in the lumbosacral cord. At the segmental level, Ib afferents provide input to several populations of interneurons as, for instance, those mediating disynaptic or trisynaptic inhibition of motoneurons (Eccles et al., 1957; for review, see Baldissera et al., 1981), and our observations of contraction-induced PAD (Lafleur et al., 1992) suggested the presence of axo-axonic synapses on Ib fibers at this level. Synaptic arrangements in Clarke's column are known to differ from those on neurons of segmental spinal reflex pathways, and it was important to confirm whether axo-axonic synapses are available in segmental pathways to modulate the synaptic actions of Ib afferents. The first aim of the present work was, therefore, to provide morphological evidence for GABA-immunoreactive axo-axonic contacts on Ib collaterals at segmental levels of the lumbosacral cord. Moreover, it is likely that the presynaptic control exerted by axo-axonic synapses is not the same when these synapses are located on afferent terminals and when they contact more proximal parts of the arborization. In this context, the second aim of our work was an attempt to analyze the distribution of axo-axonic contacts on a Ib collateral arborization.

Confocal microscopy allows an extensive screening of the intraspinal arborization of a labeled fiber, thereby optimizing the probability of detecting axo-axonic contacts. This technique was already used to demonstrate probable contacts between serotoninergic fibers and dorsal horn spinocerebellar tract neurons in 
A

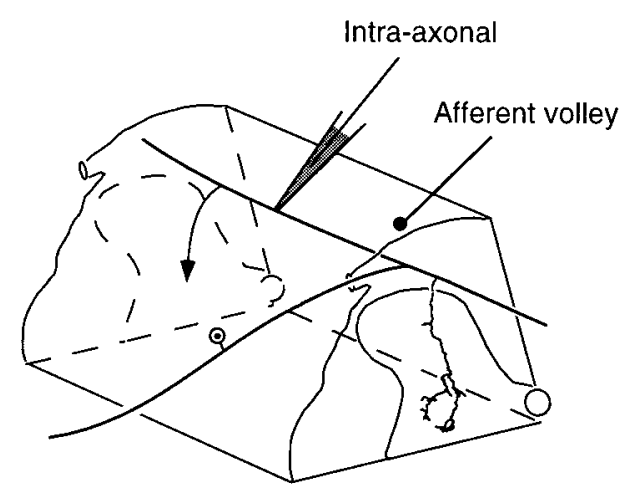

B



Figure 1. Identification of a Ib fiber from the posterior biceps semitendinosus. $A$, Schematic representation of a dorsolateral quadrant of the lumbosacral spinal cord. The Ib fiber is seen entering the spinal cord through L7 dorsal root, dividing within the dorsal column into ascending and descending branches. The single collateral drawn is the same as in Figure 3; it ramifies within the intermediate zone of the gray matter. The arrow symbolizes a second collateral. The microelectrode shows the site of intra-axonal recording. The afferent volley was recorded with a silver ball electrode at the surface of the cord dorsum near the entry of the dorsal root within the spinal cord. $B$, Superimposition of group I afferent volley (thin trace) and orthodromic action potential recorded intra-axonally (bold trace). Stimulation of PBST nerve at $2 \times$ threshold. Average of 10 successive traces. mammals (Jankowska et al., 1995). In the present study, it was used to demonstrate appositions, strongly suggesting contacts between immunostained GABAergic boutons and intraspinal terminal branches of a Ib afferent fiber labeled by a fluorescent dye.

\section{MATERIALS AND METHODS}

Preparation. Experiments were performed on adult cats $(2.9-3 \mathrm{~kg})$ anesthetized with an initial intraperitoneal dose of $45 \mathrm{mg} / \mathrm{kg}$ pentobarbitone sodium (Sagatal; May \& Baker). Additional intravenous doses $(4 \mathrm{mg} / \mathrm{kg}$ ) were delivered whenever necessary to maintain a deep level of anesthesia as assessed by: (1) miotic pupils, (2) regularity of heart rate (110-150 beats/min), and (3) stability of blood pressure $(90-130 \mathrm{mmHg}$ ). In addition, it was systematically checked that electrical stimulation of peripheral nerves at strengths eliciting potentially noxious stimuli did not modify blood pressure and heart rate.

The nerve to posterior biceps-semitendinosus (PBST) was dissected, cut, and its proximal end was mounted on a pair of electrodes for bipolar stimulation. Nerves to gastrocnemius medialis and gastrocnemius lateralis-soleus (triceps surae, TS) were dissected in continuity with their muscles and placed together on a single electrode for monopolar stimulation. TS muscles were dissected without disturbing their blood supply; their common tendon was cut and tied to an electromagnetic puller used to help functional identification of impaled afferent fibers. All other hindlimb muscles were denervated.

The lumbosacral spinal cord segments were exposed, and conventional glass micropipettes, with tips $\sim 2 \mu \mathrm{m}$ and resistance $15-20 \mathrm{M} \Omega$ when filled with a $2 \%$ solution of tetramethylrhodamine-dextran (molecular weight 3000, anionic, lysine fixable; Molecular Probes, Eugene, OR, catalog \#D-3308) in distilled water, were used for intra-axonal recording and labeling of single afferent fibers. The microelectrode was driven into the dorsal columns, close to the entry of L7 dorsal root, with an angle of $40^{\circ}$ in the caudal direction. Intra-axonal impalements occurred at depths of 0.1-0.5 mm (Fig. 1). Orthodromic activation after electrical stimulation of PBST or TS nerves ascertained the origin of the impaled fiber. Impalements were considered satisfactory when the resting membrane potential of the fiber was in the -45 to $-55 \mathrm{mV}$ range and amplitudes of orthodromic action potentials were $>70 \mathrm{mV}$. All the exposed tissues were covered with pools of mineral oil kept at $38^{\circ} \mathrm{C}$.

Identification and labeling of afferent fibers. This paper reports data collected on one Ia fiber from TS and one Ib fiber from PBST whose identification relied on the conjunction of three criteria: (1) axonal conduction velocities ( 95 and $80 \mathrm{~m} / \mathrm{sec}$ for the Ia and Ib fiber, respectively) were in the group I range; (2) in the case of the Ia fiber from TS, dynamic responses to muscle stretch or, in the case of the Ib fiber from PBST, orthodromic action potential elicited by electrical stimulation of the nerve reaching the spinal cord, together with the slow component of the group I afferent volley (Fig. 1), which suggested that it was a Ib fiber because $\sim 70 \%$ of these afferents contribute to the slow component of group I volley in PBST nerve (Laporte and Bessou, 1957); moreover, as expected for a Ib fiber, the electrical threshold for the orthodromic action potential was 1.6 times the threshold of the most excitable group I fibers; (3) on subsequent histological observation, presence (in the case of the Ia fiber) or absence (in the case of the Ib fiber, see more details in Results) of collaterals projecting to lamina IX motor nuclei (Brown and Fyffe, 1979).

Rhodamine-dextran was iontophoretically injected in the impaled fiber by passing a constant positive current of $5 \mathrm{nA}$, totaling 125 and 140 $\mathrm{nA}^{*}$ min, respectively, for the Ia and Ib fiber. The amplitudes of orthodromic action potentials elicited by electrical stimulation of the corresponding nerve were $>40 \mathrm{mV}$ throughout the injection period. The microelectrode was then withdrawn, and after a $5 \mathrm{hr}$ postinjection survival time, the animal was perfused through the descending aorta with $500 \mathrm{ml}$ of $0.1 \mathrm{M}$ PBS containing $0.1 \%$ heparin 5000 IE (Choay) and $0.1 \%$ sodium nitrite, followed by 21 of $4 \%$ paraformaldehyde in PBS at $\mathrm{pH}$ 7.4. S1-L7 spinal cord portion was removed and post-fixed for $3 \mathrm{hr}$ and then incubated for $4 \mathrm{hr}$ in a $10 \%$ sucrose solution in PBS. Parasagittal sections $(30 \mu \mathrm{m})$ were cut with a Vibratome (Leica, Nussloch, Germany).

Immunohistochemical procedures. Free-floating sections were preincubated in PBS with 20\% normal goat serum (NGS) for $30 \mathrm{~min}$ before 18 $\mathrm{hr}$ incubation in a PBS solution containing 1:400 polyclonal GABA antiserum (Biosoft) and 1\% NGS. After this primary serum incubation, the sections were rinsed and incubated in goat anti-rabbit IgG conjugated to biotin (1:100) for $75 \mathrm{~min}$. The primary GABA antiserum was revealed by incubation for $70 \mathrm{~min}$ in a solution containing fluorescein isothiocyanate (FITC)-streptavidin (1:100; Vector Laboratories, Burlingame, CA). During incubations, the free-floating sections were agitated at room temperature in solutions containing $0.3 \%$ Triton X-100. Finally, sections were washed in PBS and in distilled water, mounted in Mowiol, and stored at $4^{\circ} \mathrm{C}$ in the dark. This procedure allowed only a restricted diffusion of antibodies within the section with the consequence that GABA immunostaining was limited to a depth of 5-6 $\mu \mathrm{m}$ from the section surface. After subsequent confocal microscope observation, another cause of restriction of the examined depth was the steep attenuation of the FITC signal, which prevented the detection of GABA immunostaining from the surface of the section in contact with the slide. Selectivity of the labeling was assessed by verifying that when incubation in primary serum was replaced by incubation in PBS, FITC fluorescence was lacking.

Confocal microscopy. The two fluorochromes, tetramethylrhodamine and FITC, were independently observed using a confocal laser scanning microscope (attached to a Diaplan microscope, Leica) working with an argon-krypton laser operating in multiline mode. The tetramethylrhodamine fluorescence (red) was selectively excited with the $568 \mathrm{~nm}$ laser line and detected through a high-pass filter RG 590 (Schott). The FITC fluorescence (green) was excited with the $488 \mathrm{~nm}$ laser line and detected through an interferential narrow band filter centered at $535 \mathrm{~nm}( \pm 8 \mathrm{~nm})$ (Schott). Ten to twelve serial confocal planes at $0.5 \mu \mathrm{m}$ intervals starting from the upper surface of each section were observed through the high magnification objective $(63 \times 1.3 \mathrm{NA})$. For each confocal plane, separate tetramethylrhodamine and FITC fluorescence images were constructed by averaging four successive scannings, and subsequent numerical processing allowed fusion of tetramethylrhodamine and FITC images. In the final image the pixel size was $0.3 \mu \mathrm{m}$, and the voxel depth was $0.5 \mu \mathrm{m}$ (i.e., light was collected up to $0.25 \mu \mathrm{m}$ on both sides of the optical plane).

Three-dimensional reconstruction of a Ib collateral. Low magnification confocal images $(25 \times 0.5 \mathrm{NA}$ objective) were used for the threedimensional (3-D) reconstruction of one collateral of the Ib fiber. The 



Figure 2. A, Validation of the technique. Two Ia boutons contacting a neuron located in the ventral horn are observed on four consecutive confocal planes at $0.5 \mu \mathrm{m}$ intervals. The yellow dots are caused by close appositions between GABA-immunoreactive structures and boutons. One close apposition was observed on the four consecutive confocal planes, whereas each of the two others were visible only on two planes. Scale bar, $5 \mu \mathrm{m}$. $B, C$, Close appositions of GABA-immunoreactive structures on two Ib boutons. In each case, four consecutive confocal planes at $0.5 \mu \mathrm{m}$ intervals (pixel size 0.3 $* 0.3 \mu \mathrm{m}^{2}$ ) are shown. The arrow in $B_{1}$ points to a close apposition observed on three consecutive planes. In $C$, several GABA-immunoreactive structures in close apposition with a bouton. The star in $C$ indicates the location of a dendritic profile (not visible on confocal planes) contacted by the Ib bouton, and arrows point to GABA-immunoreactive structures. Scale bar, $5 \mu \mathrm{m}$.

rhodamine signal was systematically observed by scanning the sections with serial confocal planes at $1.5 \mu \mathrm{m}$ intervals. For each field, the confocal planes were numerically stacked to build up a 3-D block (using a modified version of NIH Image Software). Sagittal, transversal, and horizontal two-dimensional (2-D) projections of each block were then computed. The collateral was first outlined in 2-D by fitting together the corresponding projections and then sampled by a series of 550 points whose coordinates were measured on the 2-D projections. Finally, the 3-D reconstruction of the collateral was computed using these coordinates, and the location of each axo-axonic contact was assessed using the $63 \times$ images in which rhodamine and FITC signals were mixed (see above). Subsequently, the 3-D reconstruction allowed computation of an "axogram", i.e., a planar representation in which each branch of the collateral is symbolized by a vertical line whose length is equal to the branch length, and the branching points are symbolized by horizontal lines (see Fig. $3 B$ ).

Constraints of the method. The main constraint consisted in the fact that only one afferent fiber could be studied in the same experiment and that the detection of appositions of GABA-immunoreactive structures on this fiber depended on the fulfillment of five conditions. First, proper mechanical and electrical conditions had to be maintained during at least 20 min to eject a sufficient amount of tetramethylrhodamine in the fiber. Second, diffusion of the dye had to reach the terminals of the arborization. In some experiments, an apparently satisfactory ejection of the dye resulted in poor labeling of the fiber. Third, the tissue had to be well fixed. Fourth, immunolabeling (which by itself includes several steps) had to be satisfactory. Fifth, the balance between the tetramethylrhodamine and FITC intensities of fluorescence had to be compatible with confocal analysis. In total, attempts were made on eight afferents: four Ia from TS, one Ib from TS, two Ib from PB ST, and one group II from TS, but complete observations could be performed only on one Ia from TS and one Ib from PB ST.

\section{RESULTS}

\section{Validation of the method}

In a first step we labeled and examined terminal or en passant boutons of a Ia fiber, because locations, shapes, and sizes of presynaptic boutons on these fibers are well known (Conradi et al., 1983; Fyffe and Light, 1984; Pierce and Mendell, 1993). Examination of a labeled Ia fiber in confocal microscopy was aimed at (1) verifying that the method could be used to detect presynaptic appositions and (2) providing criteria for identification of these appositions.

A Ia fiber from TS was, therefore, labeled and examined for GABA-immunoreactive axo-axonic appositions. Figure $2 A$ shows four successive confocal planes with two Ia boutons (red) con- 
tacting a neuron (unlabeled), possibly a motoneuron, located in lamina IX. The location of labeled boutons showed that survival time was sufficient to allow rhodamine dextran diffusion to the most ventral endings of the arborization. The lengths of these boutons on their long axis were 7 and $2.5 \mu \mathrm{m}$, respectively, for the left and right one, i.e., in the range of the measurements made by Pierce and Mendell (1993) at the ultrastructural level. However, because the arborization of this fiber was not reconstructed, we did not know whether they were terminal or en passant boutons. GABA-immunoreactive structures (green) were present in the environment of the neuron. In addition, yellow spots of 1-1.5 $\mu \mathrm{m}$ diameter were visible on the Ia boutons (Fig. $2 A$, two spots on the left-side bouton). The yellow color indicated that both fluorochromes were detected in the same voxel, that was a volume of $0.3 * 0.3 \mu \mathrm{m}^{2}$ times the $0.5 \mu \mathrm{m}$ depth of the confocal plane (see Materials and Methods). Yellow dots were, thus, likely to reflect the presence of axo-axonic contacts on the Ia boutons (see Discussion), and the present method could, therefore, be used for detection of axo-axonic appositions on Ib fibers.

We further verified that similar yellow spots could be observed when optical planes were scanned with a higher resolution of the microscope $\left(0.15 * 0.15 \mu \mathrm{m}^{2}\right.$ pixel size $)$. We also verified that with a reduced interval between confocal planes (down to 0.08 $\mu \mathrm{m})$ the yellow zone could still be observed on several consecutive planes. But, systematic reduction of interval between confocal planes and increase in scanning resolution would have required long acquisition times and consequently long-lasting illuminations of the sections likely to induce a significant fluorescence quenching of both FITC and rhodamine. These two inconveniences were not compatible with extensive systematic searching of fiber terminals in spinal cord sections. Scanning with $0.3 * 0.3 \mu \mathrm{m}^{2}$ pixels and confocal planes at $0.5 \mu \mathrm{m}$ intervals was considered a reasonable compromise that was routinely used.

\section{Probable axo-axonic contacts on lb terminals}

In another experiment, a Ib afferent fiber from PBST was labeled after impalement at a $290 \mu \mathrm{m}$ depth from the surface of dorsal columns. Five labeled collaterals originated from the main branch travelling along the dorsal columns. They were spaced by $300-$ $1100 \mu \mathrm{m}$ in the rostrocaudal direction, and all their branches were followed to their terminals that were located exclusively within the intermediate zone of the gray matter (the trajectory of one collateral is shown in Fig. $1 A$ ). We are, therefore, confident that no collateral branch passed the intermediate zone and continued to the ventral horn, which supports our identification of the fiber as a Ib (see Materials and Methods). Close appositions between GABA-immunoreactive structures and the Ib fiber were found on all collaterals, and a detailed study of these appositions was made for two collaterals.

Figure $2 B$ shows four successive confocal planes of a Ib bouton located in the intermediate zone with a spherical GABAimmunoreactive structure of $\sim 1 \mu \mathrm{m}$ diameter (Fig. 2B, yellow spot) in close apposition. Its apposition with the bouton on three consecutive planes (Fig. $2 B_{1-3}$ ) indicated an anatomical relationship extending over at least $1.5 \mu \mathrm{m}$ within the section. In the fourth plane (Fig. $2 B_{4}$ ) the GABA-immunoreactive structure was no longer in contact with the bouton. Altogether, the shape, size, and anatomical relationship of the yellow spot strongly suggested that it corresponded to a structure making an axo-axonic contact with the Ib bouton (see Discussion). In a second example (Fig. $2 C$ ), three GABA-immunoreactive structures similarly appeared in close apposition with a Ib bouton in two successive planes (Fig. $2 C_{2-3}$ ), again suggesting that they contacted the bouton.

Using the criterion that a yellow zone must be observed on two to four consecutive confocal planes, we counted 39 and 20 GABA-immunoreactive axo-axonic appositions, respectively, for two collaterals. However, these counts were made only for the confocal planes on which GABA immunoreactions were satisfactory, i.e., within a depth of 5-6 $\mu \mathrm{m}$ (about one-fifth of the total section thickness) because of the restricted diffusion of antiGABA antiserum (see Materials and Methods). As a consequence, GABA-immunoreactive appositions could be counted on a fraction only of the collateral arborizations, and the counts were likely underestimates.

\section{Distribution of presumed axo-axonic contacts on Ib collaterals}

Figure $3 A$ shows a planar projection of the $3-\mathrm{D}$ reconstruction of the axonal trajectory of one collateral from 370 to $920 \mu \mathrm{m}$ from the dorsal border of the dorsal horn. The most ventral branches were observed in lamina VI, in keeping with Brown and Fyffe (1979). Ramifications extended in the mediolateral and rostrocaudal directions over 220 and $210 \mu \mathrm{m}$, respectively. In some very thin branches, the fluorescence was too weak to be detected by the microscope, and only varicosities were visible. The trajectory of these branches could not be followed and, therefore, these branches, their associated varicosities, and the seven appositions that were observed on these varicosities were not included in the reconstruction shown in Figure $3 A$. Thirteen appositions appearing as yellow spheres in Figure $3 A$ could be located on the collateral arborization. In total, 20 GABA-immunoreactive appositions were seen on this collateral (thirteen on the reconstruction and seven outside).

The distribution of the 13 appositions illustrated in Figure $3 A$ is shown on the axogram of this collateral (Fig. $3 B$ ). Eight were found on last-order branches at 5-94 $\mu \mathrm{m}$ distances from the end of the branch. Four of these eight were observed on swellings located in terminal positions and were suggestive of terminal boutons (a reliable identification of swellings as boutons would require to study the arborization at the ultrastructural level); the four others were also on swellings, possibly en passant boutons, located at distance from extremities. Two further appositions were located on preterminal branches at 23 and $94 \mu \mathrm{m}$, respectively, from the last-order branching point, and three were found on more proximal branches at $27-192 \mu \mathrm{m}$ from the next branching point. The two appositions pointed by arrows in Figure $3 B$ were not located on a portion of the fiber displaying clear swellings, however, we cannot rule out the possibility that they were located on small en passant boutons that went undetected with our method. All others were located on clear swellings of the fiber, suggesting en passant boutons.

The total length of the collateral was $4572 \mu \mathrm{m}$, but only a length of $1019 \mu \mathrm{m}(22.3 \%)$ was investigated for GABAimmunoreactive appositions (Fig. $3 B$, red segments). Two main branches arose from that collateral (Fig. 3B, branches 1 and 2) with respective lengths of 1761 and $1521 \mu \mathrm{m}$. Although the lengths of fiber investigated for immunoreactive GABAergic structures (red segments) were similar for branches 1 and 2 (369 and $370 \mu \mathrm{m}$, respectively), the counts of appositions were different: branch 1 received only two (i.e., 0.54/100 $\mu \mathrm{m}$ of red segments), whereas branch 2 ramifications received eight (i.e., 2.16/ $100 \mu \mathrm{m}$ of red segments). In this example, appositions were nonuniformly distributed along the collateral arborization. 
Figure 3. A, Planar projection of the threedimensional reconstruction of a Ib collateral (red) with thirteen presumed GABAimmunoreactive contacts (yellow spheres). $D, R$, and $L$ indicate, respectively, the dorsal, rostral, and lateral directions. Length of each axis, 100 $\mu \mathrm{m}$. $B$, Axogram of this collateral. The red segments indicate the segments of the fiber that were examined for GABA immunoreactivity presumed contacts in the most superficial $6 \mu \mathrm{m}$ of the sections. The pink segments indicate the remaining portions, in which GABAimmunoreactive structures were not detectable (see Materials and Methods). Further comments in Results.

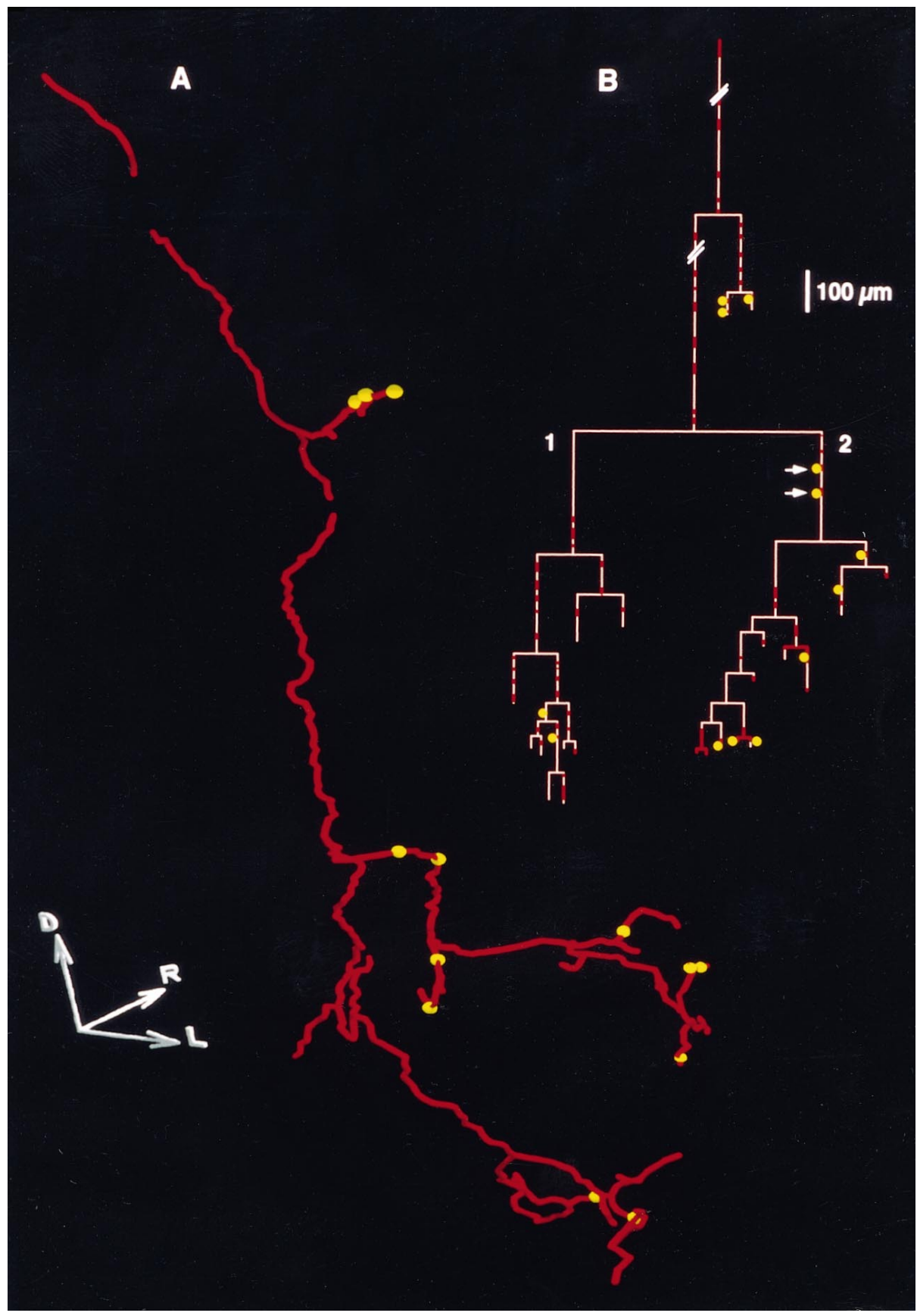

\section{DISCUSSION}

In the present study, confocal microscopy was used to detect close appositions, suggesting axo-axonic contacts, between GABAimmunoreactive structures and the intraspinal arborization of a rhodamine-filled Ib fiber in the lumbosacral cord. Altogether, 59 close appositions were observed on the arborizations of the two Ib collaterals that could be investigated in details (respectively, 20 and 39). A 3-D reconstruction of one collateral shows that the presumed contacts were nonuniformly distributed over the arborization, and mostly located on last-order branches.

Several points support the identification of the observed appositions between GABA-immunoreactive structures and Ib fiber as axo-axonic synapses, although the resolution of confocal microscope does not allow assessment of ultrastructural level features. First, apposition between the GABA-immunoreactive element and the fiber occurred in a very small element of volume $(0.3 * 0.3$ $* 0.5 \mu \mathrm{m}^{3}$ ), indicating that the GABA-immunoreactive structure was very close to the labeled fiber. Second, each apposition was observed on two to four consecutive confocal planes, indicating that it was a continuous structure extending over 1-2 $\mu \mathrm{m}$ within the depth of the section. This was confirmed by observations made with more closely spaced confocal planes $(0.25$ or $0.08 \mu \mathrm{m})$. Third, the sizes of the GABA-immunoreactive structures in the confocal plane were in the $1-2 \mu \mathrm{m}$ range, that is in keeping with the sizes $(0.5-1.5 \mu \mathrm{m})$ of the axo-axonic synapses observed at the ultrastructural level by Walmsley et al. (1987) on Ib boutons in Clarke's column. Along the same line, we observed close appositions on Ia boutons, whose sizes, shapes, and locations were similar to those of axo-axonic synapses (P boutons) observed with electron microscope (Conradi et al., 1983; Maxwell et al., 1990; 
Pierce and Mendell, 1993; Destombes et al., 1996). Finally, the GABA-immunoreactive presumed contacts were not randomly distributed over the collateral arborization (Fig. 3) as could have been the case if the appositions were simply caused by chance contacts with passing-by GABA-immunoreactive fibers.

The present work is the first demonstration of axo-axonic contacts on the segmental collaterals of a Ib fiber in the lumbosacral spinal cord. It is likely that PAD in large myelinated muscle afferents, both $\mathrm{Ia}$ and $\mathrm{Ib}$, is caused by the action of axo-axonic synapses and not by paracrine interactions, as it seems to be the case in some fine myelinated and $\mathrm{C}$ fibers carrying nociceptive information and on which axo-axonic GABAergic synapses appear to be scarce (Schmidt and Schaible, 1998).

It would, of course, be important to obtain an electron microscope confirmation of our conclusion that yellow spots do represent axo-axonic synapses of GABAergic interneurons with the labeled afferent, but this would imply overcoming methodological problems. First, tetramethylrhodamine dextran is transparent for the electron beam and another labeling method, compatible with the immunolabeling of GABA, would have to be developed. Second, it would be difficult to uncontrovertibly assign a particular contact (observed in confocal microscopy) to an axo-axonic synapse (observed with electron microscopy).

The main advantage of confocal microscopy was that it allowed an extensive and systematic screening of collateral arborizations. However, because of the restricted diffusion of anti-GABA antibody within the section, the counts of presumed axo-axonic contacts were underestimated. Our results, therefore, suggest that each collateral might be contacted by several tens of GABAimmunoreactive contacts and, because a Ib fiber usually has $>10$ collaterals in the lumbosacral spinal cord (Brown and Fyffe, 1979), the whole fiber might bear up to several hundreds of axo-axonic synapses. The present results indicate that segmental Ib collaterals possess adequate synaptic equipment to receive a significant presynaptic inhibition that might account for the filtering out of Ib input during contraction as observed in previous studies (Zytnicki et al., 1990; Lafleur et al., 1992).

The geometrical features of the Ib fiber (collateral spacings, characteristic fan-shaped arborization in laminae V-VI of the reconstructed collateral, and mediolateral and rostrocaudal extensions of this arborization) were comparable to those previously described (Hongo et al., 1978; Brown and Fyffe 1979; see also Brown, 1981). The reconstructed collateral displayed two main branches that ramified in fourth- to eighth-order branches (Fig. $3 B$ ). Most of the presumed axo-axonic contacts were found on distal branches and were ideally located to control neurotransmitter release by terminal or en passant boutons. However, two contacts (Fig. 3B, arrows) had a more proximal location on a third-order branch. Recent modeling works suggest that activation of axo-axonic synapses can reduce the amplitude of incoming action potentials by both synaptic shunt and inactivating effect of PAD on sodium current (Segev, 1990; Graham and Redman, 1994; Walmsley et al., 1995; Lamotte d'Incamps et al., 1998). If activation of axo-axonic synapses located on proximal branches induces a steep reduction of the incoming spike height, such synapses, possibly eliciting conduction failures, could control the propagation of impulses toward downstream branches. When several axo-axonic synapses are coactivated, the resulting PAD, and thereby the reduction of incoming action potentials, depends on the electrotonic structure of the arborization. Using the present morphological data (geometry of the reconstructed collateral and distribution of presumed axo-axonic contacts), a the- oretical study is currently being conducted by our group to investigate how coactivation of axo-axonic contacts could presynaptically control the information flow through the arborization.

Experiments in which the excitability of two branches of the same group I fiber were simultaneously tested by intraspinal stimulation suggest that presynaptic inhibition may differentially affect the various branches of a single arborization (Eguibar et al., 1994, 1997). This selective control of information flow could be caused by nonuniform connectivity patterns of last-order GABAergic interneurons mediating PAD with intraspinal branches of group I fibers (Eguibar et al., 1994; Quevedo et al., 1997). Depending on the distribution of the connections established by a given interneuron with branches of a single arborization, the action of this interneuron might be either restricted to some branches or widespread over the arborization. The present work does not allow us to decide whether axo-axonic contacts arose from the same or from different last-order GABAergic interneurons. However, it suggests that some branches of a $\mathrm{Ib}$ collateral bear less axo-axonic contacts than others, which might partly account for the nonuniformity of the projections of interneurons mediating presynaptic inhibition. As these interneurons are known to receive a variety of segmental and supraspinal inputs (Jankowska, 1992), their recruitment and the associated characteristics of presynaptic control of information flow might depend on the motor task.

\section{REFERENCES}

Baldissera F, Hultborn H, Illert M (1981) Integration in spinal neuronal systems. In: Handbook of Physiology, section 1, the nervous system, part II (Brookhart JM, Mountcastle VB, Brooks VB, eds), pp 509-595. Bethesda, MD: American Physiological Society.

Brown AG (1981) Organisation in the spinal cord. The anatomy and physiology of identified neurones. Berlin: Springer.

Brown AG, Fyffe REW (1979) The morphology of group Ib afferent fibre collaterals in the spinal cord of the cat. J. Physiol (Lond) 296:215-228.

Conradi S, Culheim S, Gollvik L, Kellerth JO (1983) Electron microscopic observations on the synaptic contacts of group Ia muscle spindle afferents in the cat lumbosacral spinal cord. Brain Res 265:31-39.

Curtis DR, Gynter BD, Lacey G, Beattie DT (1997) Baclofen: reduction of presynaptic calcium influx in the cat spinal cord in vivo. Exp Brain Res 113:520-533.

Destombes J, Horcholle-Bossavit G, Simon M, Thiesson D (1996) Gabalike immunoreactive terminals on lumbar motoneurons of the adult cat. A quantitative ultrastructural study. Neurosci Res 24:123-130.

Eccles JC, Eccles RM, Lundberg A (1957) Synaptic actions on motoneurones caused by impulses in Golgi tendon organ afferents. J Physiol (Lond) 138:227-252.

Eccles JC, Schmidt RF, Willis WD (1963) Pharmacological studies on presynaptic inhibition. J Physiol (Lond) 168:500-530.

Eguibar JR, Quevedo J, Jimenez I, Rudomin P (1994) Selective cortical control of information flow through different intraspinal collaterals of the same afferent fiber. Brain Res 643:328-333.

Eguibar JR, Quevedo J, Rudomin P (1997) Selective cortical and segmental control of primary afferent depolarization of single muscle afferents in the cat spinal cord. Exp Brain Res 113:411-430.

Fyffe REW, Light AR (1984) The ultrastructure of group Ia afferent fiber synapses in the lumbosacral spinal cord of the cat. Brain Res 300:201-209.

Graham B, Redman S (1994) A simulation of action potentials in synaptic boutons during presynaptic inhibition. J Neurophysiol 71:538-549.

Hongo T, Ishizuka N, Mannen H, Sasaki S (1978) Axonal trajectory of single group Ia and Ib fibres in the cat spinal cord. Neurosci Lett 8:321-328.

Jankowska E (1992) Interneuronal relay in spinal pathway from proprioceptors. Prog Neurobiol 38:335-378.

Jankowska E, Maxwell DJ, Dolk S, Krutki P, Belichenko PV, Dahlström A (1995) Contacts between serotoninergic fibres and dorsal horn 
spinocerebellar tract neurons in the cat and rat: a confocal microscopic study. Neuroscience 67:477-487.

Lafleur J, Zytnicki D, Horcholle-Bossavit G, Jami L (1992) Depolarization of $\mathrm{Ib}$ afferent axons in the cat spinal cord during homonymous muscle contraction. J Physiol (Lond) 445:345-354.

Lamotte d'Incamps B, Meunier C, Monnet M-L, Jami L, Zytnicki D (1998) Reduction of presynaptic action potentials by PAD: model and experimental study. J Comput Neurosci 5:141-156.

Laporte Y, Bessou P (1957) Etude des sous-groupes lent et rapide du groupe I (fibres afférentes d'origine musculaire de grand diamètre) chez le Chat. J Physiol (Paris) 49:1027-1037.

Maxwell DJ, Christie WM, Short AD, Brown AG (1990) Direct observations of synapses between GABA-immunoreactive boutons and muscle afferent terminals in lamina VI of the cat's spinal cord. Brain Res 530:215-222.

Pierce JP, Mendell LM (1993) Quantitative ultrastructure of Ia boutons in the ventral horn: scaling and positional relationships. J Neurosci 13:4748-4763.

Quevedo J, Eguibar JR, Lomeli J, Rudomin P (1997) Patterns of connectivity of spinal interneurons with single muscle afferents. Exp Brain Res 115:387-402.

Rudomin P (1990) Presynaptic inhibition of muscle spindle and tendon organ afferents in the mammalian spinal cord. Trends Neurosci 13:499-505.
Rudomin P, Jimenez I, Solodkin M, Duenas S (1983) Sites of action of segmental and descending control of transmission on pathways mediating PAD of Ia- and Ib-afferent fibers in cat spinal cord. J Neurophysiol 56:987-1006.

Schmidt RF, Schaible H-G (1998) Modulation of nociceptive information at the presynaptic terminals of primary afferent fibers. In: Presynaptic inhibition and neural control (Rudomin P, Romo R, Mendell L, eds), pp 424-439. New York: Oxford UP.

Segev I (1990) Computer study of presynaptic inhibition controlling the spread of action potentials into axon terminals. J Neurophysiol 63:987-998.

Stuart GJ, Redman SJ (1992) The role of GABA and $\mathrm{GABA}_{\mathrm{b}}$ receptors in presynaptic inhibition of Ia EPSPs in cat spinal motoneurones. J Physiol (Lond) 447:675-692.

Walmsley B, Wieniawa-Narkiewicz E, Nicol MJ (1987) Ultrastructural evidence related to presynaptic inhibition of primary muscle afferents in Clarke's column of the cat. J Neurosci 7:236-243.

Walmsley B, Graham B, Nicol MJ (1995) Serial E-M and simulation study of presynaptic inhibition along a group Ia collateral in the spinal cord. J Neurophysiol 74:616-623.

Zytnicki D, Lafleur J, Horcholle-Bossavit G, Lamy F, Jami L (1990) Reduction of $\mathrm{Ib}$ autogenetic inhibition in motoneurons during contraction of an ankle extensor muscle in the cat. J Neurophysiol 64:1380-1389. 